\title{
Optimization of Input Cutting Parameters on Cutting Force During Vortex Tube Jet Assisted Machining of Ti-6Al-4V.
}

\author{
Balaji. Nelge, Vinayak. Kale
}

\begin{abstract}
Titanium alloy materials machining is difficult, expensive and leads to wear and tear of the tool. The improvement in the tool life and use of optimal process parameters during machining is necessary to obtain better work piece surface finish. Here main aims to evaluate the effect of input process parameters on cutting force in Vortex Tube Jet Assisted CNC Machining of Titanium Alloy material. Taguchi L27 orthogonal array design matrix were used for experimentations by employing a Vortex Tube Jet Assisted cooling system. The significantly affecting process parameters are identified through ANOVA, and optimal parameters were identified using Taguchi and RSM. A mathematical model is proposed to estimate cutting forces based on selected input process parameters. The result reveals that the most influencing parameter is depth of cut (d). Whereas cutting speed and feed are influences very less. The cutting force (Fc) estimated from the proposed model is in close agreement with experimental results.
\end{abstract}

Keywords: ANOVA, Cutting force, Taguchi, VTJA.

\section{INTRODUCTION}

The conventional turning operations are performed using a variety of single-point cutting tools. These tools are not operated at appropriate process parameters, the tool life, and machining quality decreases whereas the cost of the product increases. To maintain the desired surface quality and tool life it is necessary to select appropriate process parameters and their range. The appropriate process parameters selection helps in reduction of cutting force (Fc) on the single-point cutting tool, many researchers highlighted the importance of cutting forces to enhance the tool life and improve surface finish.

The cutting process parameters, tool geometry, and material properties significantly influence on cutting force, quality of surface, tool wear, chip formation and cutting temperature. The Titanium alloy materials are used where elevated temperatures, high strength to weight ratio, corrosion-resistant, toughness, and biocompatibility are

\section{Revised Manuscript Received on 19 Feb 2020.}

* Correspondence Author

Balaji. Nelge*, Department of Mechanical Engineering, Rajarshri shahu college of Engineering Tathawade, Pune 411033, India. Email: balajinelge@gmail.com

Vinayak. Kale, Department of Mechanical Engineering, Indira College of Engineering \& Management, Pune 410506,India Email: vmkale@gmail.com

(C) The Authors. Published by Blue Eyes Intelligence Engineering and Sciences Publication (BEIESP). This is an open access article under the CC BY-NC-ND license (http://creativecommons.org/licenses/by-nc-nd/4.0/) required, hence it is popularly used in automotive, medical appliances, aerospace, marine, and chemical industries. Titanium alloy has been an area of research for the past few decades. The thrust research areas in the field of titanium alloy are an improvement in surface finish, machinability, tool wear resistance, reduced force in the cutting tool, thermal analysis.

Suárez, A et al. [1] studied that HPC and flood cooling face-turning of nickel-alloy 718. By using HPC more than $10 \%$ tool wear and Fc were reduced. Jafarian, G et al [2] investigated the effect of machining time on the surface roughness at the different cutting parameters by using neural network model and also implemented Non-Dominated Sorting Genetic Algorithm to optimize tool life and surface roughness. Lin et al. [3] suggest that regression analysis used for analysis the surface roughness and Fc. Zang et al [4] used MQL and MQCL cooling methods. In MQCL tool life increased by 1.57 times than MQL. Bermingham et al [5] reported that high-pressure cryogenic coolant is effective coolants for extending the tool life. Feng and Wang [6] investigated the influence of work piece hardness, f, d, Vc rake angle, and time on surface roughness by developing an empirical model. Philip S D et al [7] applied dry turning on cast DSS ASTM 955 grade5A work material. TiC and TiCN coated carbide cutting tool inserts are used. $\mathrm{S} / \mathrm{N}$ ratio and Analysis of Variance were applied to optimize the affecting parameters. Pervaiz, S et al [8] Machinability of hard material was studied to investigate the surface integrity defects, residual stresses, cracking and, microstructure characteristics using different cutting tool materials, wear mechanisms and, modes of failure. Aouic et al. [9] and Ozel et al. [10] implemented RSM to optimize the effect of cutting parameters on work piece hardness, Fc and surface roughness. Suresh et al. [11] TiC-coated tungsten carbide tool were used for machining of mild steel. RSM used for the analysis of Fc established during turning. Tao Chen et.al [12] studied the optimization of Fc on the turning of GCr15 steel by setting the optimal value of process parameters. Fratila D et.al. [13] Used Taguchi method in combination with Grey Relational Analysis with consideration of best-suited parameters like $f$, d, Vc, and tool nose radius. A. Pal et al [14] studied the influence of affecting parameter on Fc, chip tool interface temperature and surface roughness during the hard and soft turning. The effect of axial, radial and tangential forces were estimated in three different directions and revealed that the radial force is $15-20 \%$ higher than tangential force whereas axial force is higher than $102-112 \%$.

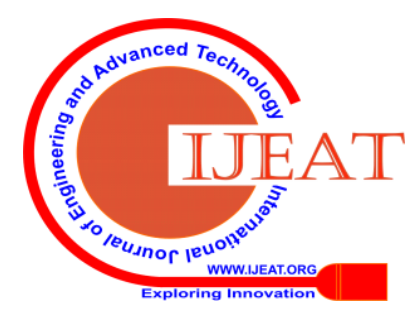


Zawada-Tomkiewicz et al [15] characterized the EN41Cr4 low chromium alloy steel was machined work piece surface through turning operation. Guo Y.B et al [16] employed the methodology to study the residual stresses produced during machining of steel whereas FEM was adopted to study the interrelationships among residual stresses, microstructures, and tool-wear.

The above literature review visualizes that researchers have carried out a study on surface finish, Fc and other performance parameters in turning operations by adopting different traditional cooling systems and coolants. However, limited literature is reported in research articles using the Vortex Tube Jet Assisted (VTJA) cooling system in combination with Ti-6Al-4V material with different cooling strategies. Therefore, the present study focuses on the effect of process parameters in concurrence with VTJA cooling systems to estimate optimal process parameters for obtaining the desired machining force and effect of surface finish.

\section{MATERIALS AND MACHINE}

The annealed Ti-6Al-4V material was selected for the study since it has good mechanical properties such as it operates at elevated temperatures, better wear-resistant, toughness, and lightweight as compared to the other materials alloys. The samples of $20 \mathrm{~mm}$ diameter $\times 100 \mathrm{~mm}$ length are considered for the study. The ceramic-based cutting tool inserts have excellent capability of machining of hardened and short chipping materials under stable conditions. The details of the triangular geometry insert (TNGA160404) specifications and geometric dimensions selected for machining are given in Fig. 1.

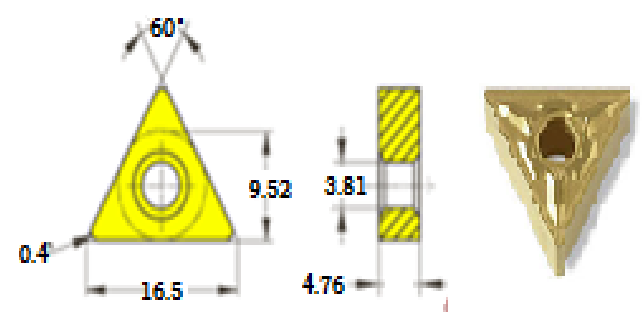

Fig 1 Cutting Tool Insert dimensions.

A VTJA cooling method is implemented during the turning of titanium alloys. The vortex tube is a part of the VTJA cooling system which was designed and developed to provide low-temperature air stream to carry heat generated during machining. This vortex tube operates over the pressures range of 2 to $5 \mathrm{~N} / \mathrm{mm} 2$. The pneumatic air pressure of $5 \mathrm{~N} / \mathrm{mm} 2$ was given input to the vortex tube and it provides $5^{\circ} \mathrm{C}$ pressurized cooled air over a cutting tool and work piece interaction region. The specifications and dimensions of the vortex tube are shown in Fig.2.

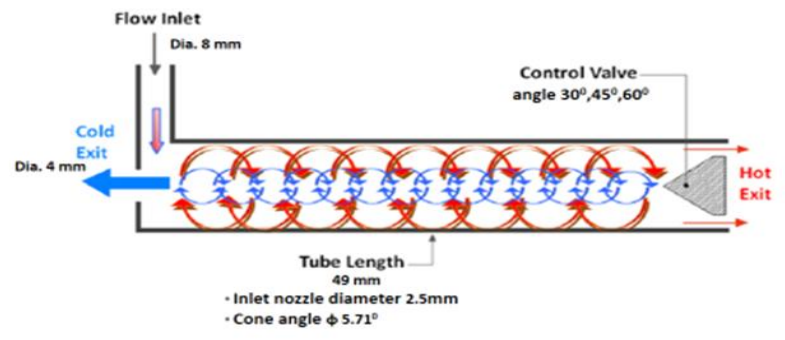

Fig.2: Schematic diagram of Vortex Tube
The vortex tube is located on TC35 Industrial type of CNC machine during experimentation is shown in Fig. 3. It operates over a spindle speed range of 50 to $3500 \mathrm{rpm}$ and a maximum power of $10 \mathrm{KW}$. The Ti-6Al-4V specimens were turned under influence of cold air stream emerging out from VTJA at a combination of different process parameters obtained from Taguchi's L27 orthogonal array design matrix. Fig. 3(a) and Fig. 3(b) show the experimental setup and work pieces machined at different combinations of process parameters during the conduction of experiments. The effect of cooling on machinating response parameters such as surface finish and machining force was assessed.

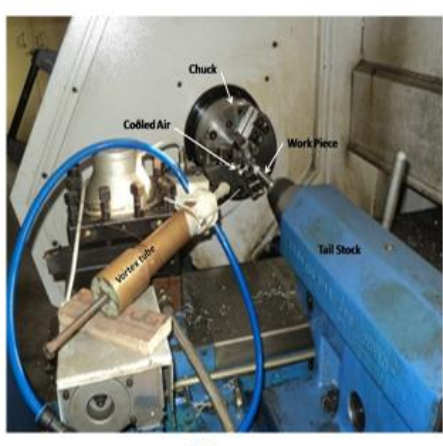

(a)

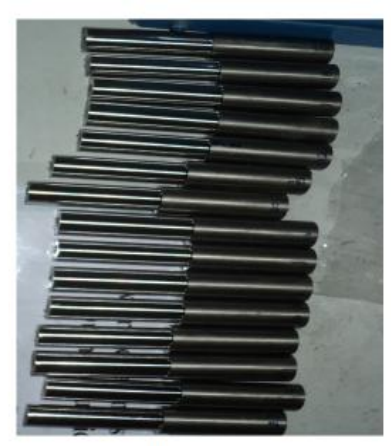

(b)
Fig.3 Experimental setup (a) VTJA Setup on CNC machine (b) Machined work pieces.

Table I: Machining parameters and their Range

\begin{tabular}{|c|c|c|c|}
\hline Factor & Level 1 & $\begin{array}{c}\text { Level } \\
2\end{array}$ & Level 3 \\
\hline Speed (Vc)(rpm) & 1000 & 1200 & 1500 \\
\hline Feed (f) (mm/rev) & 0.1 & 0.15 & 0.20 \\
\hline Depth of cut(d) (mm) & 0.5 & 1 & 1.2 \\
\hline
\end{tabular}

\section{CUTTING FORCE (FC) MEASUREMENT}

The cutting forces were measured using a three-dimensional tool dynamometer. It comprises of three independent digital display units and calibrated to display force directly. The Fc measuring unit includes self-regulating strain gauge bridges for measuring strains in three independent directions. The input process parameters and the response of the average of three tangential Fc measured using dynamometer are given in Table 2.

Table II: Cutting force measured using dynamometer

\begin{tabular}{|l|l|l|l|l|}
\hline Run & speed & feed & DOC & FC \\
\hline $\mathbf{1}$ & 1000 & 0.10 & 0.5 & 26.3333 \\
\hline $\mathbf{2}$ & 1000 & 0.10 & 1.0 & 41.6667 \\
\hline $\mathbf{3}$ & 1000 & 0.10 & 1.2 & 38.3333 \\
\hline $\mathbf{4}$ & 1000 & 0.15 & 0.5 & 28.0000 \\
\hline $\mathbf{5}$ & 1000 & 0.15 & 1.0 & 39.6667 \\
\hline $\mathbf{6}$ & 1000 & 0.15 & 1.2 & 51.3333 \\
\hline $\mathbf{7}$ & 1000 & 0.20 & 0.5 & 29.0000 \\
\hline $\mathbf{8}$ & 1000 & 0.20 & 1.0 & 50.3333 \\
\hline $\mathbf{9}$ & 1000 & 0.20 & 1.2 & 53.0000 \\
\hline $\mathbf{1 0}$ & 1200 & 0.10 & 0.5 & 15.0000 \\
\hline $\mathbf{1 1}$ & 1200 & 0.10 & 1.0 & 32.0000 \\
\hline $\mathbf{1 2}$ & 1200 & 0.10 & 1.2 & 33.3333 \\
\hline $\mathbf{1 3}$ & 1200 & 0.15 & 0.5 & 23.3333 \\
\hline $\mathbf{1 4}$ & 1200 & 0.15 & 1.0 & 37.3333 \\
\hline
\end{tabular}

Published By:
Blue Eyes Intelligence Engineering
\& Sciences Publication
(C) Copvriaht: All riahts reserved.




\begin{tabular}{|l|l|l|l|l|}
\hline $\mathbf{1 5}$ & 1200 & 0.15 & 1.2 & 43.0000 \\
\hline $\mathbf{1 6}$ & 1200 & 0.20 & 0.5 & 29.3333 \\
\hline $\mathbf{1 7}$ & 1200 & 0.20 & 1.0 & 44.3333 \\
\hline $\mathbf{1 8}$ & 1200 & 0.20 & 1.2 & 46.0000 \\
\hline $\mathbf{1 9}$ & 1500 & 0.10 & 0.5 & 15.3333 \\
\hline $\mathbf{2 0}$ & 1500 & 0.10 & 1.0 & 28.0000 \\
\hline $\mathbf{2 1}$ & 1500 & 0.10 & 1.2 & 35.0000 \\
\hline $\mathbf{2 2}$ & 1500 & 0.15 & 0.5 & 22.6667 \\
\hline $\mathbf{2 3}$ & 1500 & 0.15 & 1.0 & 39.6667 \\
\hline $\mathbf{2 4}$ & 1500 & 0.15 & 1.2 & 45.6667 \\
\hline $\mathbf{2 5}$ & 1500 & 0.20 & 0.5 & 27.6667 \\
\hline $\mathbf{2 6}$ & 1500 & 0.20 & 1.0 & 48.3333 \\
\hline $\mathbf{2 7}$ & 1500 & 0.20 & 1.2 & 57.3333 \\
\hline
\end{tabular}

\section{STATISTICAL ANALYSIS OF EXPERIMENTAL} DATA

The affecting input machining parameters like Vc, f, and d are varied as per orthogonal array L27 and the Fc was measured and analysed using MINITAB 17 software. The value of $\mathrm{S} / \mathrm{N}$ Ratio and Means obtained by considering smaller the better option [7]. The significantly affecting process parameters were ranked through ANOVA. These are given in Table 3. It also enables to perform the simultaneous test and the P-value obtained from the analysis is depicted in Table 4. The regression coefficients for Fc, Root mean-square and adjusted Root mean-square values obtained from regression analysis are $96.81 \%$ and $95.12 \%$ respectively.

Table III: Ranking of process parameters

\begin{tabular}{|c|c|c|c|}
\hline Level & Speed & Feed & Depth of Cut \\
\hline 1 & -31.71 & -28.90 & -27.39 \\
\hline 2 & -30.12 & -30.98 & -31.94 \\
\hline 3 & -30.39 & -32.33 & -32.89 \\
\hline Delta & $\mathbf{1 . 5 9}$ & $\mathbf{3 . 4 3}$ & $\mathbf{5 . 5 0}$ \\
\hline Rank & $\mathbf{3}$ & $\mathbf{2}$ & $\mathbf{1}$ \\
\hline
\end{tabular}

Table IV: ANOVA of Cutting Force

\begin{tabular}{|c|c|c|c|c|c|}
\hline Sources & DF & Adj SS & Adj MS & F-Value & P-Value \\
\hline Model & 9 & 3220.41 & 357.82 & 57.25 & 0.000 \\
\hline Linear & 3 & 2780.57 & 926.86 & 148.29 & 0.000 \\
\hline Speed & 1 & 89.10 & 89.10 & 14.26 & 0.002 \\
\hline Feed & 1 & 759.79 & 759.79 & 121.56 & 0.000 \\
\hline Depth of cut & 1 & 1942.19 & 1942.19 & 310.74 & 0.000 \\
\hline Square & 3 & 121.63 & 40.54 & 6.49 & 0.004 \\
\hline Speed*speed & 1 & 110.05 & 110.05 & 17.61 & 0.001 \\
\hline Feed*feed & 1 & 2.24 & 2.24 & 0.36 & 0.557 \\
\hline Doc*Doc & 1 & 9.35 & 9.35 & 1.50 & 0.238 \\
\hline $\begin{array}{l}\text { 3-Way } \\
\text { Interaction }\end{array}$ & 3 & 117.40 & 39.13 & 6.26 & 0.005 \\
\hline Speed*speed & 1 & 69.91 & 69.91 & 11.18 & 0.004 \\
\hline Speed*Doc & 1 & 13.33 & 13.33 & 2.13 & 0.162 \\
\hline Feed*Doc & 1 & 34.16 & 34.16 & 5.47 & 0.032 \\
\hline Error & 17 & 106.25 & 6.25 & & \\
\hline Total & 26 & 3326.67 & & & \\
\hline Sq & R-Sq & \multicolumn{2}{|c|}{ R-sq.(adj) } & \multicolumn{2}{|c|}{ R-sq(pred) } \\
\hline 2.50006 & $96.81 \%$ & \multicolumn{2}{|c|}{$95.12 \%$} & \multicolumn{2}{|c|}{$91.99 \%$} \\
\hline
\end{tabular}

The analysis of mean effect plot for means and $\mathrm{S} / \mathrm{N}$ ratio obtained from Taguchi's analysis the optimum combination of control factor for predicting minimum Fc along with the prominent control factors are shown in Fig. 4. A Smaller is the better characteristics feature was chosen for analysis to

Retrieval Number: C6240029320/2020@BEIESP

DOI: 10.35940/ijeat.C6240.049420

Journal Website: www.ijeat.org determine the optimum Fc under Vortex tube jet-assisted air cooling conditions.

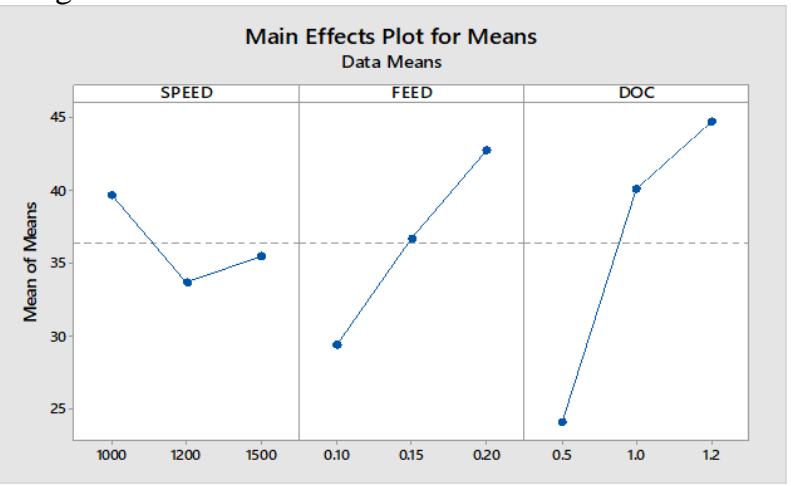

Fig: 4 Main Effect Plot for Means

\section{V.RESOPONSE SURFACE METHODOLOGY(RSM)}

The RSM statistical tool is employed to minimize the Fc and to obtain better surface finish by varying $\mathrm{Vc}$, f, and $\mathrm{d}$. In contrast to conventional methods, the RSM provides a variety of plots for the analysis of interaction among the input and output variables[9,10]. The mathematical model proposed to predict cutting force in terms of Cutting speed (S), Feed (F), and Depth of Cut (D) is given in equation (1).

$\mathbf{F c}=157.1-0.2273 S-114 F+23.1 D+0.000072 S^{\wedge} 2+244 F^{\wedge}$ $2-12.9 D^{\wedge} 2+0.1918 S F+0.01162 S D+93.6 F$ D . . (1)

\section{RESULT AND DISCUSSIONS}

Hardness is measured by micro hardness testing machine Figure show the effect of depth of cut(d) $(0.5,1.0,1.2 \mathrm{~mm})$ on hardness for all values of the spindle speeds(Vc) $(1000,1200$, $1500 \mathrm{rpm}$ ). As the depth of cut raised from 0.5 to $1.2 \mathrm{~mm}$, the hardness of Ti-6Al-V varied from $360 \mathrm{HV}$ to $415 \mathrm{HV}$ at different feed (f) $0.1,0.15$, and $0.20 \mathrm{~mm} / \mathrm{rev}$. This is as a result of lower depth of cut $0.5 \mathrm{~mm}$, at $0.1 \mathrm{~mm} / \mathrm{rev}$ with increase in Vc from 1000 to $1500 \mathrm{rpm}$ hardness also slightly increases. Only for $0.15 \mathrm{~mm} / \mathrm{rev}$ feed, increase in $\mathrm{Vc}_{\mathrm{c}}$ then hardness is decreases. When depth of cut rises from $0.5 \mathrm{~mm}$ to $1.2 \mathrm{~mm}$ then overall increasing speed and feed then hardness is decreases. Fc depends upon hardness of material [8]. For constant depth of cut, various feed and speed, hardness are measured on micro hardness tester. Which is shown in below.

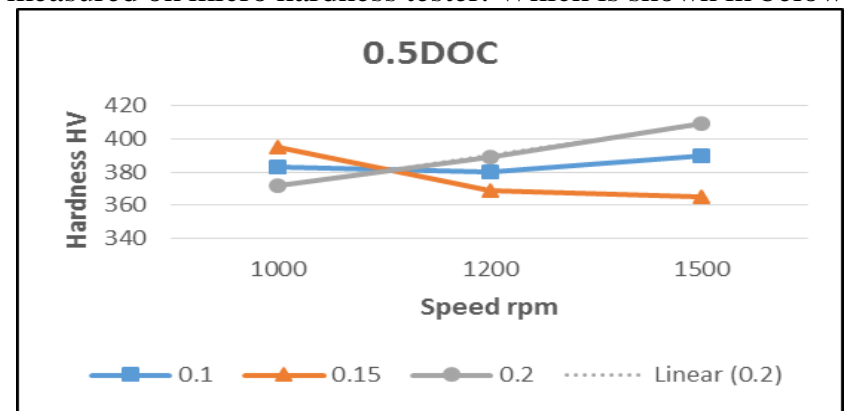

Fig: 5 Hardness variation with cutting speed for a depth of cut $0.5 \mathrm{~mm}$ at various feed

Published By:

Blue Eyes Intelligence Engineering \& Sciences Publication

(C) Copyright: All rights reserved.

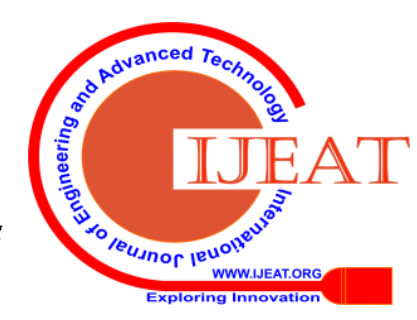




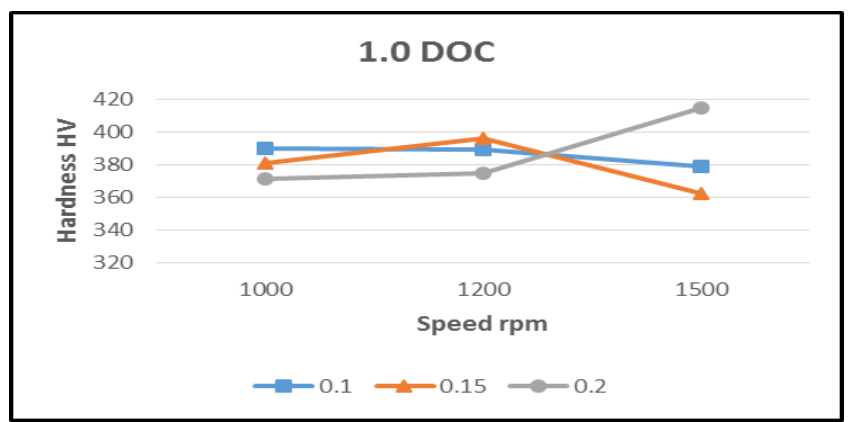

Fig: 6 Hardness variation with cutting speed for a depth of cut $1.0 \mathrm{~mm}$ at various feed

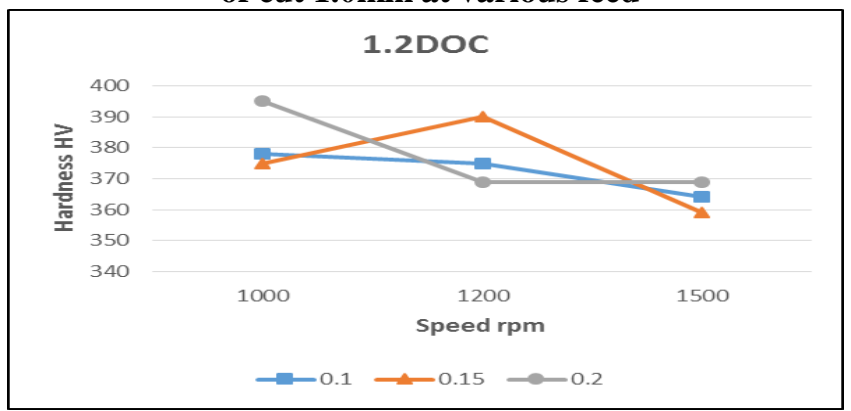

Fig: 7 Hardness variation with cutting speed for a depth of cut $1.2 \mathrm{~mm}$ at various feed.

The effect of process parameters such as Vc, f, d on Fc and surface finish has been discussed in the following section. The influential point shown in Fig. 8 affects the slope of the regression line. The residual plot shows a random distribution of data around the horizontal line in positive and negative sides, this pattern of distribution indicates that the model provides a decent fit to the data and suitable for the non-linear model. Therefore second-order polynomial equation (1) is considered for the study.

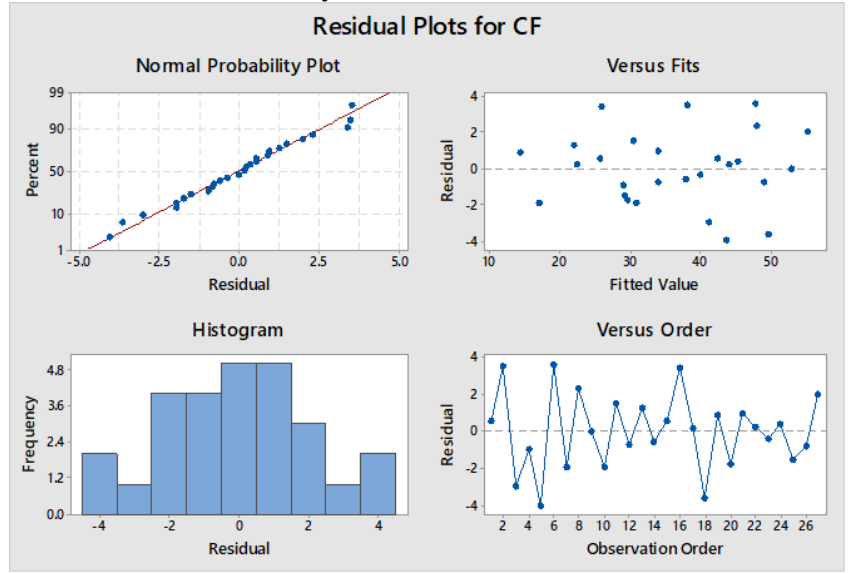

Fig. 8: Residual Plots for Cutting Force

The Lower power consumption by machine tools is observed when the machining tool is subjected to a minimum Fc. The contour plot is shown in Fig. 9 helps to predict the required Fc by referring to these contour plots. The contour plot is shown in Fig. 9(a)-Fig. 9(c) reveals the minimum Fc obtain at the feed $<0.15 \mathrm{~mm} / \mathrm{rev}$., depth of cut $<0.65 \mathrm{~mm}$. and speed in the range of 1100 to $1500 \mathrm{rpm}$. The range of process parameters selected for the study disclosed that the depth of cut plays an important role in obtaining lower Fc whereas the combination of $\mathrm{Vc}$ and $\mathrm{f}$ does not contribute more to minimizing the Fc.

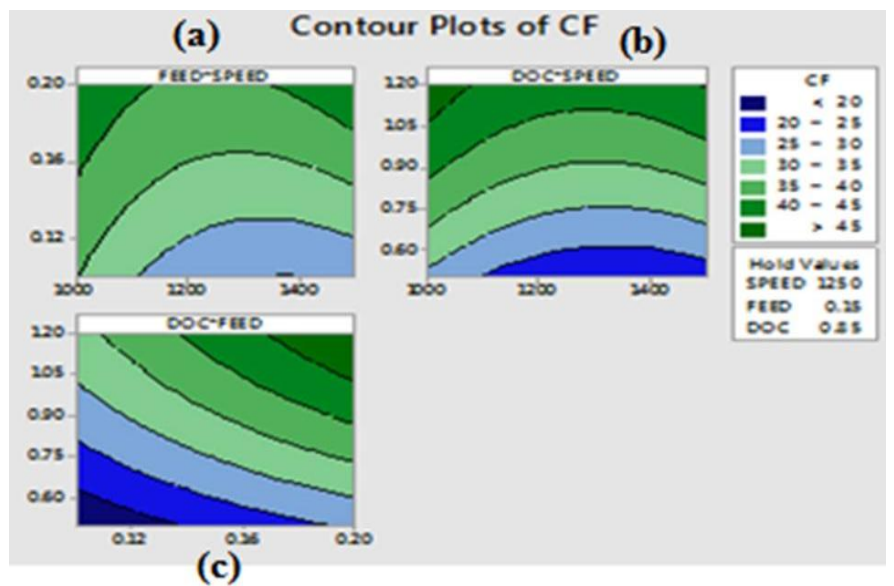

Fig 9: Contour plot represents relation between speeds, feed, Doc ad CF

The contour plot is shown in Fig. 9(a) illustrates that the Fc of 20-25 Kgf is obtained by setting speed range between 1100 rpm to $1550 \mathrm{rpm}$ and feed rate at $0.1 \mathrm{revs} / \mathrm{mm}$. However Fig. 9(b) depicts that less than $20 \mathrm{Kgf}$ is obtained at less than 0.5 mm d and 1100-1500rpm Vc. Whereas Fig. 9(c) provides a Fc lesser than $20 \mathrm{Kgf}$ for the $\mathrm{d}$ and f less than $0.65 \mathrm{~mm}$ and 0.15 $\mathrm{mm} /$ rev respectively.

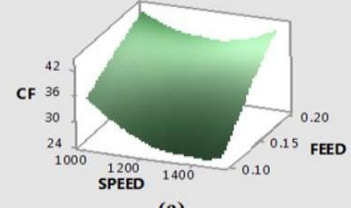

(a)

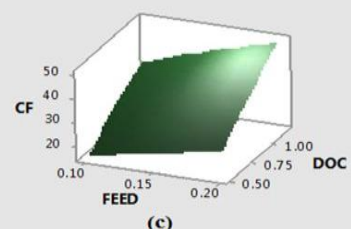

Fig. 10: Surface plot of cutting force verses speed, feed, and depth of cut

The surface plot shown in Fig.10 helps for 3D visualization of responses. This plot represents the nature of the response parameter by holding input parameters at a constant speed of $1250 \mathrm{rpm}$, 'd' of $0.85 \mathrm{~mm}$ and 'f' $0.15 \mathrm{~mm} / \mathrm{rev}$. Fig. 10(a) and Fig 10(b) reveals that the Fc raises non-linearly with an increase in $\mathrm{f}$ - Vc and Vc-d combinations respectively. However, Fig 10(c) depicts the linear variation of Fc with the increase in $\mathrm{f}$ and $\mathrm{d}$ value.

The ANOVA results are shown in Table 4 also confirm that interaction between $\mathrm{f}$ and $\mathrm{d}$ was significant for getting the desired Fc in Ti-6Al-4V Machining under VTJA cooling environment The regression model with the $\mathrm{P}$ values less than 0.002 indicate that the three cutting parameters. The most significant among all these are the $\mathrm{f}$ and $\mathrm{d}$ which is very much in bonded with the analysis of variance. In this experimentation cutting condition vortex tube cooling allows reducing the main $\mathrm{Fc}$ due to improved and near chip tool interaction. VTJA has mainly reduced the amount of heat and friction at the contact point of tool and work piece. In this experimentation optimum force obtain by using the regression equation is $17.173 \mathrm{Kgf}$.

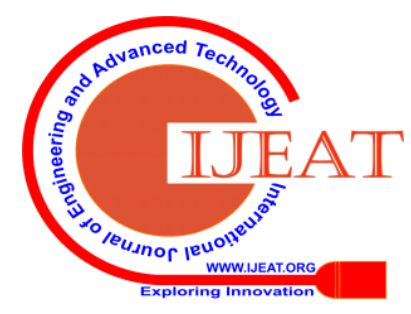


whereas by actual reading optimal Fc noted was $15 \mathrm{Kgf}$ at $0.10 \mathrm{~mm} / \mathrm{rev}$ of feed, $0.5 \mathrm{~mm}$ of the d, and 1200rpm Vc.

\section{CONCLUSIONS}

The presented research work focused on the study of the process parameters in the presence of VTJA cooling systems to estimate the optimal Fc by implementing Taguchi and RSM. The following conclusions are drawn based on experiment and statistical methods.

1. The depth of cut is the most influencing parameter on turning single point cutting tool whereas least affected by speed under VTJA. Cooling condition.

2. The proposed analytical model predicts the optimal solution within the accuracy of $12.65 \%$ as compared to the experimental results.

3. Hardness is affects to Fc, in VTJA Machining depth of cut rise from $0.5 \mathrm{~mm}$ to $1.2 \mathrm{~mm}$ then overall feed and hardness are decreases.

\section{REFERENCES}

1. Suárez, A.; López de Lacalle, L. N, Polvorosa, R.; Veiga, F.; Wretland, A. Effects of High-pressure Cooling on the Wear Patterns on Turning Inserts used on Alloy IN718.Mater.Manuf.Processes 2017,32(6),678-686.

2. Jafarian, G, Umbrello, D, Golpayegani, S.; Darake, Z. Experimental investigation to optimize tool life and surface roughness in Inconel 718 machining. Materials and Manufacturing Processes 2015; doi:10.1080/10426914.2015.1090592.

3. Lin W S, Lee B Y, Wu C L, Modeling the surface roughness and cutting force for turning”, J. Mat. Proc. Techno, 2001; 108: 286-293.

4. Zhang S, Li J F, Wang Y W, Tool life and cutting forces in end milling Inconel 718 under dry and minimum quantity cooling lubrication cutting conditions, J. Clean. Prod., 2012; 32: 81-87.

5. Bermingham MJ, Palanisamy S, Kent D, and Dargush MS. A comparison of cryogenic and high pressure emulsion cooling technologies on tool life and chip morphology in Ti-6AL-4V cutting, J. Mater. Process. Technol., 2001; 212: 2271-2285.

6. Feng C. X, Wang X, 'Development of Empirical Models for Surface Roughness Prediction in Finish Turning'. International Journal of Advanced Manufacturing Technology, 2002; 20: 348-356.

7. Philip S D, Chandramohan P, Mohanraj M, 'Optimization of surface roughness, cutting force and tool wear of nitrogen alloyed duplex stainless steel in a dry turning process using Taguchi method,' Measurement, 2014; 49:205-215.

8. Pervaiz, S.; Rashid, A.; Deiab, I.; Nicolescu, M. Influence of tool materials on machinability of titanium- and nickel-based alloys: a review. Materials and Manufacturing Processes 2014, 29 (3), 219-252. doi:10.1080/10426914.2014.880460.

9. Aouici H., Yallese M.A, Chaoui K., et al, 'Rigal analysis of surface roughness and cutting force components in hard turning with CBN tool: prediction model and cutting conditions optimization', Measurement, 2012; 45: 344-353.

10. T.Ozel, T.K. Hus, E. Zerne, 'Effects of cutting edge geometry, work piece hardness feed rate and cutting speed on surface roughness and forces in finish turning of hardened AISIH 13 steel', Int. J. Adv. Manuf. Technol. 2005; 25: 262-269.

11. Suresh R., Basavarajappa S., Gaitonde V.N., Samuel G.L, 'Machinability investigations on hardened AISI 4340 steel using coated carbide insert', Int. J. Refractory Metals and Hard Materials, 2012; 33: 75-86.

12. Tao C, Suyan L, Bangxin H, Guangjun L, 'Study on cutting force and surface micro-topography of hard turning of GCr15 steel', Int. J. Adv. Manuf. Technol., 2014;72:1639-1645.

13. Fratila D, Caizar C, 'Application of Taguchi method to selection of optimal lubrication and cutting conditions in face milling of ALMg3', J. Clean. Prod., 2011; 19: 640-645.

14. Pal A, Choudhry S.K, Chinchanikar S, 'Machinability Assessment through Experimental Investigation during Hard and Soft Turning of Hardened Steel', Procedia Materials Science, 2014; 6: 80-91.
15. Anna Z T, 'Analysis of surface roughness parameters achieved by hard turning with the use of PCBN tools', Estonian Journal of Engineering, 2011; 17: 88-99.

16. Guo, Y.B.; Li, W.; Jawahir, I.S. 'Surface integrity characterization and prediction in machining of hardened and difficult-to-machine alloys; a state-of-the-art research review and analysis'. Machining Science and Technology 2009, 13, $437-470$.

\section{AUTHORS PROFILE}

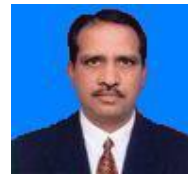

Balaji Nelge. He has completed the M.E in Mechanical Engineering, Department of Mechanical Engineering, He has 09 Journal and 06 conference papers. His major fields of studies are Manufacturing process, His research interest is in the hard material machining, optimization and developing processing for super alloys ( $\mathrm{Ti} \& \mathrm{Ni}$ materials.).

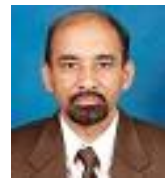

Vinayak Kale, $\mathrm{PhD}, 24$ Journal and 18 conference papers. Main area of interest are heat transfer and operation research.

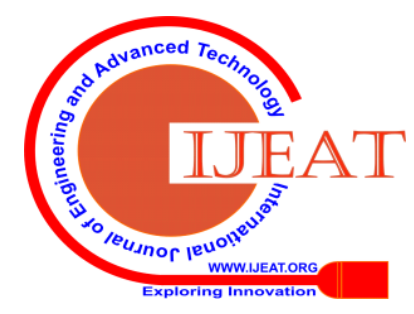

